\title{
Recurrent Cushing's Syndrome Manifesting as Pseudotumor Cerebri with Isolated Cranial Nerve Six Palsy
}

Tarek El-Halabi and Johnny Salameh*

Department of Neurology, American University of Beirut Medical Center, Cairo Street, Hamra, Beirut, Lebanon

\begin{abstract}
Pseudotumor cerebri has been described after exposure to steroids as well as following steroid withdrawal, causing adrenocortical insufficiency resulting in reduction of cerebrospinal fluid absorption and leading to intracranial hypertension. In our case, the patient had increased intracranial pressure caused by hypocortisolism. Cases of pseudotumor cerebri after resection of an ACTH-secreting adenoma has been reported in the literature, more often in children than in adults, and mostly occurring after steroid taper compared to steroid excess states. Our case highlights the importance of considering recurrence of Cushing's syndrome in adults presenting with isolated cranial nerve palsy and pseudotumor cerebri.
\end{abstract}

Keywords: Cranial nerve six palsy; Pseudotumor cerebri; Cushing's syndrome

\section{Introduction}

Pseudotumor cerebri is diagnosed by the presence of headache, papilledema, and elevated cerebrospinal fluid pressure ( $>20 \mathrm{~cm}$ water) in the absence of abnormalities in the level of consciousness, findings on neurological examination (except for cranial nerve paresis), neuroimaging, or cerebrospinal fluid analysis [1]. This has been reported following the reduction in the dose of maintenance steroids, as well as in some cases after surgery for Cushing's disease resulting in noticeable decrease in endogenous cortisol production which was observed mainly in children [1].

The pathophysiology of the cranial nerve palsies in Idiopathic Intracranial Hypertension remains poorly understood. Abducens nerve palsy can be explained by nerve traction from increased pressure, with a high likelihood of it being compressed as it exits Dorello's canal under the petroclinoid ligament [2]. Isolated cranial nerve six palsy is defined as a pure lateral rectus weakness with no evidence to suggest a specific etiology [3]. It is the most common isolated cranial nerve palsy due its long peripheral trajectory [2]. However, in rare cases of adult onset unilateral cranial nerve six palsy in the absence of papilledema, headache, pulsatile tinnitus, or transient visual obscuration, the diagnosis of pseudotumor cerebri remains very challenging [4].

\section{Case Report}

A 29-year-old right handed lady, with a history of pituitary microadenoma status post 4 surgeries and bilateral adrenalectomy, with no recurrence, presented with horizontal binocular diplopia noticeable mainly on left lateral gaze of 1-week duration. Upon further questioning, she reports a headache of three months duration, intermittent, bilateral, more on the left side. She also reports few pounds weight gain but denied any congestion or lacrimation. She had a lumbar puncture at another institution that showed elevated opening pressure but normal studies following which she reported improvement in her symptoms.

On physical examination, patient was afebrile with a blood pressure of $131 / 68 \mathrm{mmHg}$ and a heart rate of 91 beats per minute. Neurologic examination was as follows: Pupils were equal, round, and reactive to light. Funduscopic examination revealed clear optic discs. No papilledema, no decrease in visual acuity. Visual fields were normal to double stimulation and confrontation. No ptosis was noted.
Extraocular movements were examined and on primary gaze, the left eye was slightly adducted. On horizontal gaze toward the left, the left eye had limited abduction, and the diplopia became more pronounced. Diplopia improved when looking at near objects vs. far objects. There was no restriction of patient's gaze toward the right, superiorly, or inferiorly. There was no evidence of internuclear ophthalmoplegia or nystagmus. The right eye had full extraocular movements. Remaining cranial nerves examined were all normal including a symmetric face, V1-V3 sensation, hearing, gag reflex, midline tongue, and shoulder shrug. No other focal neurologic deficits were noted.

Patient was maintained on steroid replacement therapy for 2 years after her last microadenoma surgery; however, 6 months prior to her presentation the dose was slightly reduced. She was on a daily dose of hydrocortisone $10 \mathrm{mg}$, prednisone $10 \mathrm{mg}$, and Fludrocortisone 0.25 mg. Results of the lumbar puncture were unremarkable. Magnetic Resonance Imaging of the brain with venous phase was also normal. Her ACTH level was $464 \mathrm{pg} / \mathrm{ml}$ (Normal range 0 - 46 pg/ml). After our evaluation and work up, she was started on Acetazolamide $250 \mathrm{mg}$ twice daily and then increased to $500 \mathrm{mg}$ twice daily. In addition, the daily doses of hydrocortisone, prednisone, and fludrocortisone were all doubled. One month later, the patient had marked improvement in her diplopia and headache. A repeat lumbar puncture was done which showed an opening pressure of $9 \mathrm{~cm} \mathrm{H}_{2} \mathrm{O}$ with normal Cerebrospinal fluid cell count, cytology showed increased lymphocytes but there were no malignant cells.

\section{Discussion}

In adults, the most likely etiology of isolated sixth nerve palsy is ischemic mono-neuropathy [3] but in middle-aged patients, other etiologies, such as increased intracranial pressure, multiple sclerosis,

*Corresponding author: Johnny Salameh, Department of Neurology, American University of Beirut Medical Center, Cairo Street Hamra, Beirut, Lebanon, Tel: 9611350000; E mail: johnnysalameh@hotmail.com

Received February 19, 2018; Accepted March 24, 2018; Published March 28, 2018

Citation: El-Halabi T, Salameh J (2018) Recurrent Cushing's Syndrome Manifesting as Pseudotumor Cerebri with Isolated Cranial Nerve Six Palsy. J Neurol Disord 6: 378. doi:10.4172/2329-6895.1000378

Copyright: (c) 2018 El-Halabi T, et al. This is an open-access article distributed under the terms of the Creative Commons Attribution License, which permits unrestricted use, distribution, and reproduction in any medium, provided the original author and source are credited. 
Citation: El-Halabi T, Salameh J (2018) Recurrent Cushing's Syndrome Manifesting as Pseudotumor Cerebri with Isolated Cranial Nerve Six Palsy. J Neurol Disord 6: 378. doi:10.4172/2329-6895.1000378

meningitis, and neurodegenerative diseases should be ruled out [1]. Prospective studies have shown serious pathology in 63\% of Abducens nerve palsy patients, with even higher percentage in patients younger than 50 years of age. As such, a sixth nerve palsy in this age group always warrants extensive work up including neuroimaging and lumbar puncture.

Our patient is an unusual case of an adult with Cushing's syndrome which was operated 3 times; and on the last $\left(4^{\text {th }}\right)$ surgery no pathology was found. She was then started on steroids for 2 years, and after a slight taper in her steroids doses 6 months prior to her presentation, she developed idiopathic intracranial hypertension with prominent isolated left cranial nerve six palsy and a mild headache. The patient had major improvement in her diplopia and headache after draining CSF, steroid replacement, and acetazolamide.

\section{Conclusion}

Pseudotumor cerebri has been described after exposure to steroids as well as following steroid withdrawal, causing adrenocortical insufficiency resulting in reduction of cerebrospinal fluid absorption and leading to intracranial hypertension. In our case, the patient had increased intracranial pressure caused by hypocortisolism. Cases of pseudotumor cerebri after resection of an ACTH-secreting adenoma has been reported in the literature, more often in children than in adults, and mostly occurring after steroid taper compared to steroid excess states. Our case highlights the importance of considering recurrence of Cushing 's syndrome in adults presenting with isolated cranial nerve palsy and pseudotumor cerebri.

\section{References}

1. Kiehna NE, Keil M, Lodish M, Stratakis C, Oldfield HE (2010) Pseudotumor cerebri after surgical remission of Cushing's disease. J Clin Endocrinol Metab 95: 1528-1532.

2. Brinar VV, Habek M, Ozretic D, Djakovic V, Matijevic V (2007) Isolated nontraumatic abducens nerve palsy. Acta Neurol Belg 107: 126-130.

3. Azarmina M, Azarmina $\mathrm{H}$ (2013) The six syndromes of the sixth cranial nerve. J Ophthalmic Vis Res 8: 160-171.

4. Quattrone A, Bono F, Fera F, Lavano A (2006) Isolated unilateral abducens palsy in idiopathic intracranial hypertension without papilledema. Eur J Neurol 13: $670-671$. 